COMMISSION No. 20

\title{
POSITIONS AND MOTIONS OF MINOR PLANETS, COMETS AND SATELLITES \\ (POSITIONS ET MOUVEMENTS DES PETITES PLANETES, \\ DES COMETES ET DES SATELLITES)
}

Report of Meetings on 4th, 8th and 10th of August, 1988

PRESIDENT: Y. Kozai

SECRETARY: D.K. Yeomans

\section{ADMINISTRATION AND SCIENTIFIC SESSION ON OCCULTATION(Aug. 4)}

The President welcomed members of the Commission to Baltimore. Noting that the Commission's Vice-President, Yu.V. Batrakov, requested that his name not be put in nomination for President he requested and received Commission's confirmation of R.M. West as President and A. Carusi as Vice-President for the coming triennium. D.K. Yeomans was confirmed as the secretary without objection. All present stood in silence for a few moments in remembrance of the Commission members who had died during the triennium: Y.-Z. Zhang and W.H. Robertson.

E. Bowell requested the establishment of a Working Group for Minor Planet Citations. This group would be responsible for preparing and maintaining a machine readable file of minor planet names that would include the citations and references. Bowell also suggested that the Group look into the possibility of awarding a suitable diploma to accompany future citations. Members of the Organizing Committee, new Commission members and consultants, as well as the members of various Working Groups were confirmed during the second administrative session on August 10. J.D. Mulholland renewed his plea that Commission members consider the advantages of converting from the current sexagesimal system to one based entirely upon decimal fractions.

As chairman of the Occultation Working Group, R. Millis gave a report of activities during the triennium. Predictions for sixteen occultations and their ground tracks were distributed to the network of observers in 1987 with the corresponding number for 1988 being thirteen. Two notable successes were the December 8, 1987 occultation by 324 Bamberga and the June 9, 1988 occultation by Pluto. Chords from some thirteen sites in China, Japan and in the USA allowed an accurate size and shape to be determined for Bamberga. Evidence for the presence of atmosphere on Pluto was one of the preliminary results from observations at seven sites in Australia, New Zealand and from the Kuiper Airborne Observatory. Ten upcoming occultation events were then reviewed. Following the December 29, 1988 occultation of minor planet 6 Hebe, the 1989 events include 87 Sylvia(Feb. 28), 324 Bamberga(Mar. 18), 4 Vesta(April 2), Saturn(July 3), 9 Metis(Aug. 6), 216 Kleopatra(Aug. 14), 4 Vesta (Aug. 19), 521 Brixia(Oct. 23), and 1 Ceres(Nov. 11).

A discussion then followed with Millis noting a very limited number of observers outside the U.S. with portable telescopes suitable for occultation work and E. Roemer noting the relatively few observatories(i.e. Lick and Lowell) with plate 
scale and field of view to provide reliable last minute refinements to occultation predictions. D. Dunham noted that Bordeaux Observatory was also capable of making accurate position updates, and with the northern hemisphere PPM catalogue now available, observatories having instruments with narrow fields of view can also participate in position improvements. Dunham and L. Kristensen both stressed that the occultation community should single out the most important events and distribute the predictions as soon as possible. Millis resigned as chairman of the Working Group and after some discussions on the role of the Group, Commission members took straw votes to confirm that the Working Group on Occultations should remain in operation and that L. Wasserman should become the chairman.

\section{SCIENTIFIC SESSION: MINOR PLANET(August 4)}

The director of the Minor Planet Center, B.G. Marsden, noted that the proposal, introduced at the time of the "Asteroid II" colloquim in March 1988, concerning the processing of discovery observations of main-belt minor planets, is already having a beneficial effect. This means that real time discoveries are credited only when accurate positions are provided on two or more nights. Processing of singlenight reports, which have typically involved 60 percent of all discoveries of minor planets, is now deliberately being delayed for several months. This change is already tending to make the astrometric database more useful for orbit and identification work and to credit discoveries to the more useful and systematic observers. While exceptions can be made, the same procedure is also recommended for non-visual discoveries of comets and Earth-approaching minor planets.

There is also value in obtaining two positions(rather than one or three) on each night and in also applying the two-night rule with regard to observations of known minor planets, particularly numbered objects or those on the critical list. Observers are strongly encouraged to provide magnitude for new discoveries and objects about whose identification they made be uncertain. Particular care should be taken in recording the UT time of observation since an extraordinarily large number of observations are reported to the Minor Planet Center with the wrong date! After the first two nights of observations, the observer needs to decide whether further follow-up is necessary. The possibility of rapid communication by electronic mail now means that the Minor Planet Center can advise the observer whether a discovery is really new. Observers should try to arrange follow-up on two further nights such that the total arc spans two dark runs, or at least ten days during a single dark run. What is not wanted are observations on just three nights over less than a five-day span. The Minor Planet Center would also find it helpful if an observer would send all the observations of a particular object during one dark run in no more than two communications. If identifications do not show up when the arc approaches one month, further observations will be needed to secure recovery at another opposition. Extended coverage of this type, yielding arcs of up to 90 days and more, is particularly desirable if any kind of follow-up is to be given to very faint objects, for which past identifications are rather unlikely.

V.K. Abalakin briefly reported on activities at the Institute for Theoretical Astronomy. A few copies of the 1989 edition of Efemeridy Malyky Planet were available at the meeting. Beginning with this edition, there are some substantial improvements in the standard opposition ephemerides. Although the total amount 
of space occupied is unchanged, the ephemerides are now given with greater precision, there is more specific information on accuracy, and there are more detailed data on heliocentric and geocentric distances and phase angle.

E. Bowell described the Lowell Observatory, U.K. Schmidt Telescope Asteroid Survey(LUKAS). This survey is designed to provide astrometric data for minor planets(spanning more than 60 days) in an effort to reduce the biases in the distribution of orbital elements introduced by observational selection effects on discoveries. With a magnitude limit of approximately 22 , this survey will utilize automatic scanning and measurement procedures to obtain minor planet positions on wide field photographic plates.

T. Gehrels described the continuing SPACEWATCH program at Steward Observatory whereby a CCD detector on a $0.9 \mathrm{~m}$ telescope is used in a scanning mode to efficiently collect positional data on minor planets and comets. The $320 \times 512$ element CCD detector is currently being replaced by a much larger 2048x2048 one.

E. Helin discussed the plans for a recently acquired $1.2 \mathrm{~m}$ telescope at the Jet Propulsion Laboratory's Table Mountain Observatory. This instrument will be used to make observations on targets of opportunity and to follow up on discoveries made with over-subscribed telescopes at Palomar Mountain and elsewhere. This facility, which should be operational in approximately six months, will augument the minor planet and comet astrometric programs that are active at Oak Ridge, Steward, Palomar, Lowell and elsewhere.

Brief communications were also presented by $H$. Debehogne regarding the use of the $0.4 \mathrm{~m}$ GPO telescope at La Silla for astrometric observations and by $\mathrm{R}$. Rajamohan regarding the recent plans to use a $0.46 \mathrm{~m}$ Schmidt to develop the skills required to make and reduce minor planet and comet observations. Rajamohan outlined future plans that include the use of a larger instrument to search for minor planets, comets and planet X. This initiative was welcomed by Marsden as the first astrometric program in India.

A substantial part of the session was devoted to presentations related to the proposed transfer of Commission 20 activities from B1950.0 to J2000.0. On introducing this part, Marsden reminded members that the Commission had in 1983 specifically deferred action on this matter until suitable star catalogues and charts became widely available. Following the publication of the FK5, it appeared that significant progress in this area was now being made.

C. Smith described the recent completion of a SAO type catalogue that has been put onto the FK5 system at the U.S. Naval Observatory. Schwan's regional corrections have been applied and the Aoki/Standish conversion procedures have been used to transform the positions to the epoch of J2000.0.

S. Roeser described the Positions and Proper Motions(PPM) reference star catalogue for the northern hemisphere. A total of 181581 stars are included on the FK5 system and the entire catalogue is available on the J2000 epoch. Approximately six published positions were used to determine proper motions, and the stellar RMS position error, at present epoch, is approximately 0.3 arcsec. A preliminary version is already available on tape and the final version will be ready 
by the end of 1989; it will then be delivered to the National Space Science Data Center at the Goddard Space Flight Center. With the completion of the CPC2 a recent epoch star catalogue is available for the southern hemisphere. Roesner expressed the desire to complete a PPM catalogue for the southern hemisphere if the Astrographic Catalogue could be made available in machine readable form.

J. Russel provided information about the completed Space Telescope Guide Star Catalogue. Containing 20 million stars, this catalogue covers the entire sky down to mag. 15 at the galactic poles and mag. 13 at the galactic equator. It is on the FK5-J2000 system and has a mean plate epoch of 1983. However, the catalogue position errors can reach 2-3 arc seconds when positions were measured in the corners of the Schmidt plates. No proper motion information is given. The primary mode of distribution of this catalogue will be on 3-4 compact optical disks.

It was necessary also to consider procedures for the conversion of existing B1950.0 observations and orbital elements of minor planets and comets to J2000.0 and the availability of appropriate representations of the solar and planetary coordinates. Marsden pointed out that, while the formulations on page B42 and B43 of the current Astronomical Almanac might be appropriate for conversions between B1950.0 and J2000.0 in the case of stars, the artificially induced proper motion(arising mainly from the change in the precession constant) created a potential problem with positions of minor planets and comets.

E.M. Standish discussed the numerical DE200 planetary ephemeris and P.K. Seidelmann remarked that the U.S. Naval Observatory was preparing a floppy disk planetary ephemeris over the interval 1800-2050 based upon JPL's DE200. The precision of the available data will be at the milli-arcsecond level. Standish recommended that the existing angular elements for comets and minor planets that are approximately referred to the FK4-B1950 system be transformed to the FK5-J2000 system by the application of a simple coordinate rotation such as that given on page S37 of the Astronomical Almanac for 1984. This would presumably be a stopgap procedure until new orbital elements could be diretly determined using observations in the J2000 system. More sophisticated conversion procedures should be applied for transforming FK4-B1950 observations to FK5-J2000. Marsden showed an example, in which the different treatment of observations and orbits(including the solar coordinates) yielded residuals in the two systems that typically differed by 0.5 arcsec. It was proposed that an ad-hoc committee be formed to examine further the requirements and to specify schedules and procedures for the transformation.

E. Tedesco outlined the new procedures for predicting minor planet magnitude data. He noted that the $H$ and $G$ values given in the IRAS documentation refer to blue magnitudes while those published in $M P C 11096$ refer to visual magnitudes, the latter values for $G$ being constrained to be in the range $0.0-0.5$.

Lack of time prompted the postponement of Z. Knežević's paper on proper elements and families to the minor planet session of Commission 15. L.K. Kristensen presented a brief report on the lost minor planet, 719 Albert, noting that a reinvestigation of available data shows that they are consistent if due account is taken of the uncertainties in the times of the end-points of the photographic trails. The asteroid's orbital position is currently indeterminate so that search ephemerides cannot be computed to aid recovery. 


\section{SCIENTIFIC SESSION: COMETS(August 8)}

E. Roemer remarked, in taking the chair, that the past triennium was an extremely successful one that included the flyby of six spacecrafts past comet Halley in March 1986 and a record number of comet discoveries. C. Shoemaker was credited with her 14th discovery and W. Bradfield with his 13th.

S. Nakano reported upon the activities of Japanese amateur astronomers in observing and identifying minor planets. Over three thousand astrometric observations of minor planets have been reported by Japanese amateur astronomers from 30 separate observatories. Kobayashi identified 40 recently observed minor planets with known objects, while Nakano has 37 such identifications to his credit. Marsden praised the several contributions of Japanese amateur astronomers to minor planet research and noted a parellel record with respect to observations of comets.

On behalf of E.M. Shoemaker, E. Bowell presented a request for comet position and magnitude observations at large heliocentric distances. Shoemaker's recent results for the flux of long period comets at Jupiter's orbit is 3-4 times his previous estimates. This result suggests that estimates for the number of comets in the Oort cloud should be increased and that this increased flux will affect the deduced comet cratering rates for the planets and their satellites. The revised numbers may also be in better accord with the observed population of short period comets.

B.G. Marsden gave a brief summary of the status of astrometric observing program at Oak Ridge. Although considerable thought had been given a few years ago to utilizing a mosaic of small CCD detectors and inclinometers to perform the astrometry, lack of a suitable computer interface had made it impossible to put the system into practice. With a large CCD now due in the near future, it seems appropriate to make use of it directly, without the need for inclinometers. In the meantime, the observing is still done photographically. When the program with the $1.5 \mathrm{~m}$ reflector started in 1972 it was sometimes possible to reach objects of mag. 20, but the steadily brightening sky now makes it difficult to reach fainter than mag. 18. Nevertheless, even if recoveries of comets rarely take place at Oak Ridge, final observations and other critical positions are still frequently obtained and rapidly reduced. In the area of minor planets, it is noteworthy that 30 percent of the minor planets are newly numbered on the basis of Oak Ridge observations alone at their latest oppositions.

Roemer then noted that comets with poor observation histories are a problem to recover using the small fields of CCD-equipped telescopes. A request was made for retaining the capabilities of some wide field instruments for recovering poorly observed objects.

S. Ostro discussed the use of radar Doppler and range observations as astrometric data types for comets and minor planets to be used to refine their mathematical models and observatory positions. Radar data exist for 52 minor planets and 5 comets. Radar data are powerful astrometrically because they have fractional precision between $10^{-5}$ and $10^{-8}$ and they are made along the line-of-sight, and hence are orthogonal to optical, angular position measurements. Line-of-sight velocities can be measured to the sub $\mathrm{mm} / \mathrm{s}$ level. The power of the radar data, when used in conjunction with optical data, is particularly evident when the optical 
data arcs are small. Ostro suggested that when the radar data are published, the transmit frequency, received time, round trip time delay and Doppler frequency shift be given rather than derived quantities such as distance and velocity.

Z. Sekania outlined his recent work on the dynamics of comets. The momentum transfered to a comet by gas outflowing from the discrete centers of activity on its nucleus depends upon their outgassing pattern and surface distribution, and on the comet's spin vector. As a rule, seasonal effects are dominant and the momentum distribution is highly asymmetrical with respect to perihelion. This results in a major contribution from the transferred momentum's radial component to the perturbation of the comet's mean motion. On the other hand, the role of a rotation lag(A.M. vs. P.M. activity) is reduced, especially for high-obliquity objects. For comets like Encke with a high equatorial obliquity, there may be no correlation between nucleus spin direction and orbital acceleration or decceleration.

D.K. Yeomans showed recent results in modelling nongravitational effects in the orbit determination studies for the comets, d'Arrest, Giacobini-Zinner, Halley and Kopff. By allowing the peak in the water vaporization curve to move to either side of perihelion in the nongravitational force model, the orbital fit is improved and the optimum offset of the water vaporization curve corresponds to the similar offset of greatest intrinsic brightness in the comet's light curve. This work also supports the notion that nongravitational effects are due primarily to a radial thrust acting asymmetrically with respect to perihelion rather than to the traverse thrust component.

Marsden remarked on the matter of availability of ephemerides. A quick poll showed that, in spite of the existence of the ephemeris-computation feature in the Central Bureau for Astronomical Telegrams/Minor Planet Center computer service and the widespread distribution of suitable computer programs, more than one-third of those present still required printed ephemerides. While the frequent publication of the IAU Circulars and $M P C$ s is clearly appropriate for ephemerides for newlydiscovered comets, the need for observers to plan ever further into the future made it desirable to enquire whether annual sources of ephemerides, such as the BAA, OAA, and ICQ Handbooks, which principally include data for the predicted returns of periodic comets, could be published farther in advance. In response to a request from the Nautical Almanac Offices, orbital elements for periodic comets are now provided in the $M P C$ s several years ahead, with those for all the comets returning in 1991 already published in $M P C$ s dated May $1,1988$.

M.E. Baily presented a short paper by himself and C.R. Stagg entitled "Cratering constraints on the Oort cloud inner core." A re-analysis of the relative proportion of craters produced by different types of Earth crossing bodies shows that active comets probably account for less than 10 percent. This considerably relaxes cratering constraints on models of the inner Oort cloud. The absence of evidence for large surges of comet showers suggests that the inner Oort cloud, if it exists at all, does not have to be as dense as once thought.

A. Carusi gave an update on the Long Term Evolution Project. The original long-term numerical integrations for 132 comets were complete for comets through December 1984. The first update of the catalogue will include orbits for 14 new comets in addition to 23 orbits that have been recently improved. These results 
should be completed by the end of 1988 and released by mid-1989.

Yeomans then reported on the successful campaign of the Astrometry Network of the International Halley Watch. For comet Giacobini-Zinner a total of 1300 astrometric observations(Jan. 28, 1984 - March 31, 1987) have been received from 81 observatories. The corresponding numbers for comet Halley are 6631 observations(Oct. 16, 1982 - Feb. 14, 1988) from 143 observatories. For Giacobini-Zinner, 83 percent of all data were less than three times the RMS residual and hence used in orbital solutions; for Halley 90 percent of the received data were used. All data for comets Giacobini-Zinner and Halley will be archived and made available on compact disks and, if resources allow, as printed volumes.

P. Weissman brought the Commission up-to-date on NASA's proposed rendezvous mission to a comet with an enroute flyby of a main-belt minor planet. The mission currently under consideration would launch in August 1995, fly by minor planet 449 Hamburga in January 1998 and effect a rendezvous with comet Kopff in August 2000. The spacecraft would investigate the comet throughout its perihelion passage in December 2002 with cameras and other instruments that include a nucleus penetrator to investigate its subsurface ices.

R. Farquhar summarized plans to use existing and future spacecrafts to investigate comets using a low budget approach. The Japanese spacecrafts, Sakigake and Suisei, are scheduled for Earch swingbys in 1992 with the former craft then being targeted for comet Honda-Mrkos-Pajdušáková in 1996 and the latter being targeted for comet Giacobini-Zinner in 1998. Using an Earth swingby, ESA's Giotto spacecraft can be targeted for comets Grigg-Skjellerup or Hartley 2 if upcoming tests indicate that sacecraft's camera is operational. Farquhar also mentioned studies that are underway by NASA and ISAS(Japan) to assess the possibilities of a comet coma sample return mission in the late 1990's.

\section{SCIENTIFIC SESSION: SATELLITES(August 8)}

J.-E. Arlot, chairman of the Working Group on Satellites, began the session by proposing two resolutions to make more accessible the satellite astrometric data taken by spacecrafts. The complete text of these internal resolutions follows:

(1) The Working Group on Natural Satellites of Commission 20 recommends that NASA make available to all investigators the positional observations of the satellites of the planets that have been made from spacecraft. Preferably, these data should be made available in the form of right ascensions and declinations together with necessary auxiliary data such as the position of the spacecraft at the observation time. The Viking and Mariner observations of Phobos and Deimos that have been prepared in support of the Soviet Phobos mission are excellent examples of what should be done to make this type of data available.

(2) Commission 20 recommends that its Working Group on Natural Satellites study the creation of an international center of astrometric data on the natural satellites. Under the auspices of the IAU, this center would work to make these data widely available to researchers in this area of study. 
Arlot then read an abstract of a paper by V. Shor on the refinements of the orbits of Phobos and Deimos using ground-based and spacecraft observations. Together with the Viking spacecraft data, optical data over the interval 1877-1976 were used to determine the satellites' orbits.

J.R. Rohde reported that an almanac for many of the natural satellites of all the planets from Mars through Pluto was nearly complete. Rectangular coordinates and differential positions with respect to the planet will be available on floppy disks over the interval 1988-2000 from the U.S. Naval Observatory within a few months. Arlot then reported that $M$. Chapron-Touzé and he had produced compact ephemerides of the Galilean satellites of Jupiter on floppy disks. Rohde described his work on the outer satellites of Jupiter noting that there are systematic positive biases for the early right ascension observations of JVI and JVII.

K. Aksnes outlined the recent work of the Satellite Nomenclature Liaison Committee with regard to the Uranian satellites that were discovered by the Voyager 2 spacecraft. These ten faint satellites, all located inward of Miranda, were named after Shakespearean characters and numbered from the outermost inward. The agreed upon names and designations have been published on the IAU Circular 4609 dated June 8, 1988.

B. Morando delivered a short paper by J. Laskar and R.A. Jacobson on the five large Uranian satellites. Laskar's analytic theory agrees well with numerical integration results with differences of only $12-100 \mathrm{~km}$ over the ten year interval. The actual uncertainty of their theory is of the order of $100-250 \mathrm{~km}$ in position. Their work has recently appeared in the Astronomy and Astrophysics, and an ephemeris is available on floppy disk.

D. Pascu reported upon the U.S. Naval Observatory's program of CCD observations of faint satellites using the $1.5 \mathrm{~m}$ astrometric reflector at Flagstaff, Arizona. They employ a 2 to 1 re-imaging coronagraph and a 800x800 CCD. Observations of Miranda over the interval 1981-1985 have already been published. Photometry $(B$, $V$ ) of the faint satellites is a useful by-product of the astrometric positions.

Morando then reported upon a program to observe the mutual eclipses of the Galilean satellites of Jupiter. He noted the important role of amateur astronomers who have been making observations with photoelectric detectors as well as visual observations. Two-dimensional photometry has been done with vido cameras. Future infrared observations would be useful to detect hot spots on satellite surface.

\section{ADMINISTRATION II(August 10)}

Y. Kozai prepared the lists of the Commission's proposed new members and consultants and of the members and chairmen of the various Working Groups. These groups, listed below, were first reviewed and then approved by the Commission members.

New members: M. Chapront-Touzé, J.R. Donnison, G. Dourneau, J.A. Fernandez, W. Ferreri, Miao-fu He, H. Kinoshita, Z. Knežević, D. Lazzaro, P.J. Message, A. Milani, T. Pauwels, D.A. Ramsay, 
E.M. Shoemaker, M. Soma, E.M. Standish, D. Taylor, D.J. Tholen, Z. Vavrova, A. Whipple, M. Yuasa

Consultants: C.M. Bardwell, C. Blanco, S.J. Bus, K.I. Churyumov, R.W. Farquhar, R.A. Robinson, E. Kazimirchak-Polonskaya, H. Kosai, R.H. McNaught, S.M. Milbourn, Z.M. Pereyra, V. Protitch-Benishek, N. Samojlova-Yakhontova, T. Seki, C.S. Shoemaker, J.B. Tatum

Working Group on Comets: M.E. Bailey, N.A.Belyaev, M.P. Candy, A. Carusi, A. Gilmore, L. Kresák, B.G. Marsden, S. Nakano, H. Rickman(ch.), E. Roemer, G. Sitarski, R.M. West, P. Wild, D.K. Yeomans

Working Group on Occultations: J.C. Bhattacharyya, C. Blanco, G.L. Blow, D.W. Dunham, Miao-fu He, A.R. Klemola, L.K. Kristensen, R.L. Millis, M.D. Overbeek, V. Shor, M. Soma, G.E. Taylor, L. Wasserman(ch.)

Working Group on Satellites: K. Aksnes, J.-E. Arlot(ch.), S. Ferraz-Mello, P.A. Ianna, R.A. Jacobson, J.H. Lieske, B. Morando, J.D. Mulholland, T. Nakamura, D. Pascu, M. Rapaport, P.K. Seidelmann, V. Shor, D.B. Taylor, R. Vieira-Martins

Satellite Nomenclature Liaison Committee: K. Aksnes(chairman \& delegate to WGPS), J.-E. Arlot, A. Carusi, R.M. West, P.K. Seidelman(Vice-chairman $\&$ alternate delegate to WGPS)

Minor Planet Names Committee: A. Carusi, B.G. Marsden, R.M. West

Comet Nomenclature Committee: B.G. Marsden, H. Rickman, R.M. West

Standing Committee to Oversee Publication of Photometric Data for Minor Planets: E.L.G. Bowell, A. Harris, B.G. Marsden, R.M. West

Representative to Working Group on Reference Systems: D.K. Yeomans

Study Group on the Origins of Minor Planet Names: V.K. Abalakin,

E.L.G. Bowell(ch.), F. Edmondson, H. Haupt, L.K. Kristensen, B.G. Marsden, P. Millman, J.D. Mulholland, E. Roemer, L. Schmadel, K. Tomita,

I. Van Houten-Groeneveld, J.X. Zhang

The Commission then considered an appeal by T. Gehrels concerning a minor planet name proposed by him but rejected by the Minor Planet Names Committee as having current political implications. It was noted that the appeal had been properly filed in accord with the resolution adopted by the Commission at the XIXth General Assembly(Trans. IAU XIXB, p. 185, 1986). A secret ballot by Commission members was requested, and the decision of the Minor Planet Names Committee was sustained by the vote of 31 to 6 . The straw poll of the Commission on their desire to convert from the sexagesimal to the decimal system showed that most were opposed to the change at this time. 
Marsden then gave a report on the ad hoc committee on coordinate conversions. The committee introduced the following internal resolution:

Noting that progress is being made toward the general availability of necessary star catalogues, Commission 20 recommends that steps be taken to convert from B1950.0 to J2000.0 in the near future. For this purpose, an ad hoc committee of Commission 20 is hereby established to prepare for the transition, with appropriate procedures to be presented for endorsement at the next General Assembly. The target date for the change to the system of FK5 on the standard equinox J2000.0 is 1992 January 1 . This requires that the work of the Committee be completed by 1990 June 30 .

For the Committee to discharge its duties, the Commission notes that adequate star catalogues must be available by the above target date. In this context, an adequate catalogue means one whose stellar density is at least as great as that of the SAOC. The Commission recognizes that machine-readable versions will satisfy many users, but notes that printed versions and associated charts are still desirable. Special effort is clearly needed to achieve the stated goal for the southern hemisphere. Commission 20 urges that groups engaged in the compilation of such catalogues be supported adequately to ensure that these tasks come to fruition in a timely manner.

Additionally, the transition requires protocols for the conversion of both observations and orbital elements between the existing and the new systems, as well as for the determination of orbits and the calculation of ephemerides in the new system. The committee shall be responsible for formulating these procedures, giving attention to their internal coherence and reversibility, insofar as this be possible.

The ad hoc Committee is to be known as the System Transition Committee, and its chairman shall be the Commission's representative to the IAU Working Group on Reference Systems.

This resolution and the following members of the System Transition Committee were reviewed and approved by the Commission members present:

T.E. Corbin, P.D. Hemenway, L.K. Kristensen, K. Hurukawa,

B.G. Marsden, J.D. Mulholland, S. Roesr, P.K. Seidelmann, V. Shor,

C.A. Smith, E.M. Standish, R.M. West, D.K. Yeomans(ch.)

C.S. Kay, representing Commission 22, read a proposal to set up a working party to gather opinion and formulate recommended policy concerning the pollution of interplanetary space by spacecraft exhausts and solid debris. Commission 20 voiced its support of the initiative and nominated Y. Kozai and R.M. West as its representatives on the working party.

The Commission adjourned its final session to reconvene in three years at the next General Assembly in Buenos Aires, Argentina. 